\title{
Magnetic structure and crystalline electric field effects in the triangular antiferromagnet $\mathrm{CePtAl}_{4} \mathrm{Ge}_{2}$
}

\author{
S. Shin $\odot,{ }^{1}$ V. Pomjakushin, ${ }^{2}$ L. Keller, ${ }^{2}$ P. F. S. Rosa, ${ }^{3}$ U. Stuhr, ${ }^{2}$ C. Niedermayer, ${ }^{2}$ R. Sibille, ${ }^{2}$ S. Toth, ${ }^{2}$ J. Kim,${ }^{1,4}$ H. Jang, \\ S.-K. Son, ${ }^{1}$ H.-O. Lee, ${ }^{1}$ T. Shang $\odot,{ }^{5}$ M. Medarde, ${ }^{5}$ E. D. Bauer, ${ }^{3}$ M. Kenzelmann, ${ }^{2,{ }^{*}}$ and T. Park ${ }^{1, \dagger}$ \\ ${ }^{1}$ Center for Quantum Materials and Superconductivity (CQMS) and Department of Physics, Sungkyunkwan University, \\ Suwon 16419, South Korea \\ ${ }^{2}$ Laboratory for Neutron Scattering and Imaging, Paul Scherrer Institut, CH-5232, Villigen PSI, Switzerland \\ ${ }^{3}$ Los Alamos National Laboratory, Los Alamos, New Mexico 87545, USA \\ ${ }^{4}$ Institute of Basic Science, Sungkyunkwan University, Suwon 16419, South Korea \\ ${ }^{5}$ Laboratory for Multiscale Materials Experiments, Paul Scherrer Institut, CH-5232 Villigen PSI, Switzerland
}

(Received 3 September 2019; revised manuscript received 28 April 2020; accepted 26 May 2020; published 15 June 2020)

\begin{abstract}
Kondo metal CePtAl ${ }_{4} \mathrm{Ge}_{2}$ exhibits long-range antiferromagnetic order below $2.3 \mathrm{~K}$. Neutron powderdiffraction experiments reveal that Ce moments order antiferromagnetically with an incommensurate ordering wave vector $\boldsymbol{k}=(1.39,0,0.09)$. Inelastic neutron powder scattering experiments show a magnetic excitation at $14.5 \mathrm{meV}$, which corresponds to the first excited state due to the crystalline electric field splitting of the ground-state multiplet of $\mathrm{Ce}^{3+}$. The temperature and field dependence of the magnetization of $\mathrm{CePtAl}_{4} \mathrm{Ge}_{2}$ is consistent with a doublet ground state with a dominant $\left|j_{\mathrm{z}}=1 / 2\right\rangle$ character and a first excited doublet $\left|j_{\mathrm{z}}=3 / 2\right\rangle$ at $14.5 \mathrm{meV}$.
\end{abstract}

DOI: 10.1103/PhysRevB.101.224421

\section{INTRODUCTION}

Magnetic frustration in metallic systems may induce remarkable emergent ground states, including nonmagnetic quantum spin liquids and chiral magnetic structures hosting anomalous Hall effect [1]. As Wannier proposed in 1950, geometrically frustrated triangular lattices with Ising moments cannot satisfy all antiferromagnetic couplings, giving rise to magnetic frustration [2]. In the past half century, condensed-matter physics witnessed a flourish of theoretical and experimental research on frustrated insulators [3-6]. More recently, the interplay between magnetic frustration and metallic degrees of freedom started to be investigated [7-9], and distinct frustration characteristics emerge due to the presence of itinerant electrons [10]. For the case of insulators, the magnetic moments vary their direction to avoid frustration and form a magnetically ordered/disordered state with exotic quantum magnetic properties, such as the quantum spin-liquid state [6]. In a metallic system, however, the magnetic moments may vary their amplitude as well as their direction [10]. In particular, magnetic frustration in $f$-electron metallic systems has been a promising route for the discovery of novel ground states. Experimentally, frustrated magnetic structures have been reported in a breadth of $f$-electron compounds including CePdAl [7,11], $\mathrm{UNi}_{4} \mathrm{~B}$ [12], YbPtIn [13], and $\mathrm{YbAgGe}[13,14]$. Magnetic frustration also gives rise to a skyrmion lattice in $\mathrm{Gd}_{2} \mathrm{PdSi}_{3}$ [15], an electronic nematic state in $\mathrm{CeRhIn}_{5}$ [16-18], and field-/pressure-induced

\footnotetext{
*michel.kenzelmann@psi.ch

†tp8701@skku.edu
}

quantum criticality in CePdAl [19-22], CeAgGe [23-25], and CeRhSn [26-28]. Several theoretical works proposed that these electronic states in $f$-electron metallic systems could be ascribed to the interplay between the Ruderman-KittelKasuya-Yosida (RKKY) interaction, the Kondo effect, and the strength of the magnetic frustration [29-31].

The recently reported Ce-based antiferromagnet CePtAl ${ }_{4} \mathrm{Ge}_{2}$ is an ideal candidate to study the interplay among the competing orders because it crystallizes in the trigonal structure $(R \overline{3} \mathrm{~m})$ and shows a metallic behavior [32]. Electrical resistivity revealed a local minimum at 18 $\mathrm{K}$ due to the Kondo effect and a maximum at $6 \mathrm{~K}$ due to the onset of the Kondo coherent state. The magnetic $\mathrm{Ce}^{3+}$ ions form a two-dimensional triangular lattice and order antiferromagnetically (AFM) below $T_{\mathrm{N}}=2.3 \mathrm{~K}$, with in plane being the magnetic easy plane. Magnetic specific-heat capacity shows an anomaly at $T_{\mathrm{N}}$ and the magnetic entropy recovers only $61 \%$ of $R \ln 2$ due to Kondo screening effects and reduced dimensionality of the magnetism. The Kondo temperature is estimated as $2 \mathrm{~K}$ - the temperature where the magnetic entropy recovers $0.5 R \ln 2$. This is similar to the situation in CePdAl, where the geometrical frustration of $\mathrm{Ce}$ moments is responsible for the mixed ordered state with incommensurate ordering wave vector $\boldsymbol{k}=(0.5,0, \sim 0.35)$ [7]. Also in $\mathrm{CePtAl}_{4} \mathrm{Ge}_{2}$, the competing orders favored by RKKY interactions, the Kondo effect, and metallicity in the presence of magnetic frustration are expected to induce emergent quantum states near a $T=0 \mathrm{~K}$ AFM phase transition.

Here we determine the magnetic structure of the triangular antiferromagnetic metallic compound $\mathrm{CePtAl}_{4} \mathrm{Ge}_{2}$ via 
neutron-diffraction measurements. Elastic neutron-scattering experiments showed that $\mathrm{Ce}$ moments order antiferromagnetically below $2.3 \mathrm{~K}$ with an incommensurate ordering wave vector $(1.39,0,0.09)$ in the $R \overline{3} m$ space group. Rietveld analysis of the magnetic Bragg pattern associated with this ordering wave vector and relevant basis of magnetic moments reveals that the magnitude of the magnetic moments is modulated along the crystallographic [100] and [001] directions. Inelastic neutron scattering as well as crystalline electric field (CEF) fits of the anisotropic magnetic properties revealed that the ground state is a doublet with dominant $\left|j_{\mathrm{z}}=1 / 2\right\rangle$ in the $J=5 / 2$ multiplet and the first excited CEF level at $14.5 \mathrm{meV}$ is composed of the $\left|j_{\mathrm{Z}}=3 / 2\right\rangle$ states.

\section{EXPERIMENT}

Single crystals of $\mathrm{CePtAl}_{4} \mathrm{Ge}_{2}$ were grown by an $\mathrm{Al}_{0.73} \mathrm{Ge}_{0.27}$ self-flux method [32]. About $4 \mathrm{~g}$ of single crystals were gently crushed to fine powder for neutron powder experiments. The magnetic structure of $\mathrm{CePtAl}_{4} \mathrm{Ge}_{2}$ was studied using the high-resolution powder diffractometer for thermal neutrons, HRPT [33], and the Cold Neutron Powder Diffractometer, DMC [34], at the Swiss Spallation Neutron Source SINQ at the Paul Scherrer Institut (PSI) in Villigen, Switzerland. Neutrons with wavelength of $\lambda=1.886 \AA$ and $\lambda=2.46 \AA$ were used for HRPT and DMC, respectively. The neutron powder diffraction patterns were collected at 10 and $1.7 \mathrm{~K}$ at DMC with the powder loaded in a vanadium container. For the low-temperature experiment at $0.07 \mathrm{~K}$ on DMC, the same powder was transferred to a copper container. The resulting powder diffraction patterns were refined by the Rietveld software package FULLPROF suite [35] and the symmetry analysis was performed with the BasiReps program and software tools from the Bilbao crystallographic server [36]. A typical orange cryostat was used for temperature control above $1.5 \mathrm{~K}$, while dilution refrigerator was used for temperature control below $1.5 \mathrm{~K}$.

Single-crystal neutron-diffraction experiments were performed on a single piece of single crystal with mass of 74 $\mathrm{mg}$ and dimension of $4 \times 3 \times 1 \mathrm{~mm}^{3}$ using two different instruments, the cold neutron triple-axis spectrometer RITA-II and the thermal neutron diffractometer Zebra at SINQ. The single crystal was aligned with the $(h, h, 0)$ reciprocal plane horizontally on RITA-II with wavelength $(\lambda)$ of $4.05 \AA$ and $(h$, $0, l)$ reciprocal plane horizontally on Zebra with $\lambda=2.32 \AA$. The cold neutrons with $\lambda$ of $4.05 \AA$ at RITA-II were produced by the (002) Bragg reflection of a vertically focusing pyrolytic graphite (PG) monochromator. The higher harmonics were minimized with a collimator $\left(\alpha=80^{\prime}\right)$ and a nitrogen-cooled Be filter. The scattered neutron diffraction was recorded by the nine-bladed PG analyzer that provides high-resolution data. On Zebra, the 2.32-Å neutrons were produced by the (002) Bragg reflection of a vertically focused PG monochromator. For both instruments, the sample holder was attached to the mixing chamber of an Oxford dilution refrigerator in order to monitor the magnetic diffraction down to $0.1 \mathrm{~K}$.

Inelastic neutron-scattering experiments on a 4-g powder sample loaded inside a vanadium container inserted in the orange cryostat and were performed at 10 and $120 \mathrm{~K}$ on the thermal neutron triple-axis spectrometer, EIGER, at SINQ.

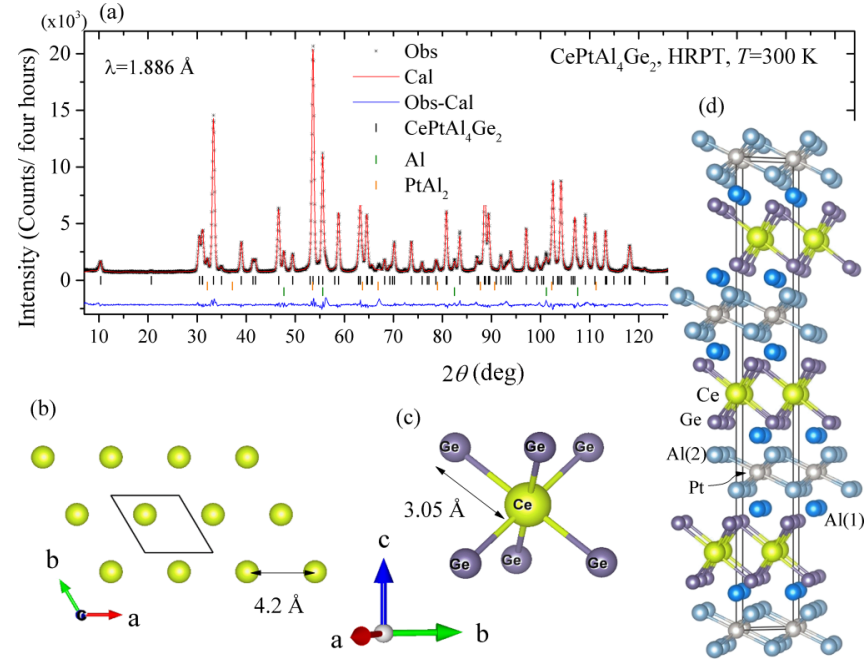

FIG. 1. Neutron powder-diffraction pattern of $\mathrm{CePtAl}_{4} \mathrm{Ge}_{2}$ at room temperature and its crystal structure. (a) Neutron powderdiffraction pattern of $\mathrm{CePtAl}_{4} \mathrm{Ge}_{2}$ was measured on HRPT with wavelength of $1.886 \AA$ at room temperature. Black symbols are the experimental data and the red line is the result of Rietveld refinement. The whole pattern is explained by trigonal symmetry $(R \overline{3} m$, SG 166) of $\mathrm{CePtAl}_{4} \mathrm{Ge}_{2}$ with small impurities of $\mathrm{PtAl}_{2}(F m \overline{3} m$, SG 225) and $\mathrm{Al}(F m \overline{3} m$, SG 225$)$. (b) Triangular closely packed layer with Ce-Ce distance of $4.2 \AA$ is separated by $10.38 \AA$ with in-plane shift from the equivalent Ce layer. (c) The six $\mathrm{Ge}^{4+}$ ions that are the first nearest neighbors surrounding the $\mathrm{Ce}^{3+}$ ion, which split the $J=5 / 2$ multiplet into three doublet states. (d) Crystal structure of $\mathrm{CePtAl}_{4} \mathrm{Ge}_{2}$ with a large $c / a(=7.6)$ ratio unit cell.

Inelastic spectra as a function of transferred energy were measured at various $\mathbf{Q}$ with fixed final energy of $E_{\mathrm{f}}=14.7 \mathrm{meV}$ at 10 and $120 \mathrm{~K}$. Magnetization data were obtained using a superconducting quantum interference device (SQUID) in a Quantum Design magnetic property measurement system (MPMS-XL, from Quantum Design Inc.). The magnetization was measured using a single piece of $\mathrm{CePtAl} \mathrm{Ge}_{2}$ with mass of $8 \mathrm{mg}$ as a function of temperature from 1.8 to $300 \mathrm{~K}$ under the applied magnetic field of $5 \mathrm{kOe}$ along the [100] and [001] crystallographic directions. We note that the magnetic susceptibility measured for the large, high-quality single crystal is different from that reported in Ref. [32], which emphasizes the importance of a large signal-to-background ratio (see details in Ref. [37]). Magnetic field dependence of the magnetization was measured at $10 \mathrm{~K}$ up to $70 \mathrm{kOe}$.

\section{RESULTS}

Figure 1(a) displays the neutron powder-diffraction pattern measured at HRPT with $\lambda=1.886 \AA$ at room temperature. The pattern was best refined by a trigonal, $R \overline{3} m$ (space group 166), crystal symmetry with the unit cell of $a=$ 4.19128(9),$c=31.77224(70) \AA$, and $\gamma=120^{\circ}$. Some Bragg peaks that are inconsistent with the $R \overline{3} m$ symmetry were successfully refined by introducing two additional $\mathrm{PtAl}_{2}$ and $\mathrm{Al}$ phases, whose symmetry is $F m \overline{3} m$ (space group 225 ) in both cases. The amount of secondary phases is only a few percent of the total intensity and none of them were reported 


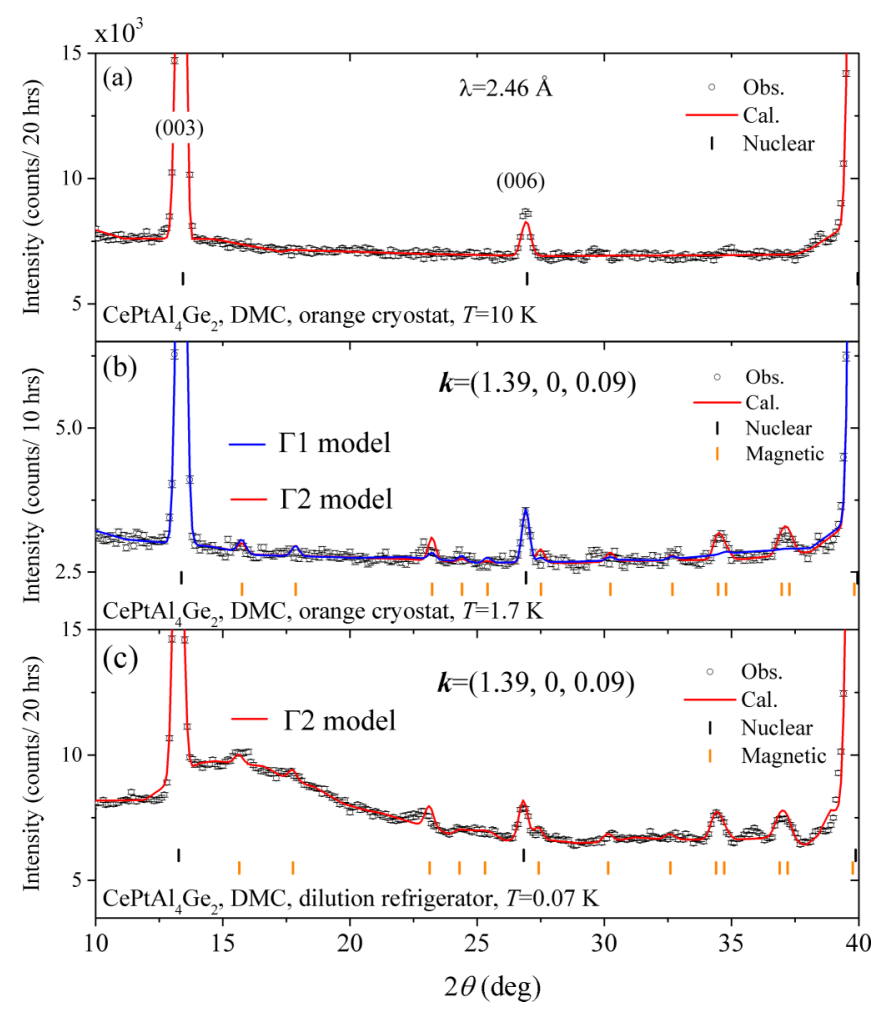

FIG. 2. Rietveld refinement results on neutron powder diffraction pattern of $\mathrm{CePtAl}_{4} \mathrm{Ge}_{2}$. Neutron powder-diffraction pattern of $\mathrm{CePtAl}_{4} \mathrm{Ge}_{2}$ measured at $10 \mathrm{~K}(\mathrm{a}), 1.7 \mathrm{~K}$ (b), and (c) $0.07 \mathrm{~K}$. Black open circle, red line, black bar, and orange bar indicate observed neutron intensity, calculation, nuclear Bragg position of $R \overline{3} \mathrm{~m}$, and magnetic Bragg position of the ordering wave vector, $\boldsymbol{k}=$ $(1.39,0,0.09)$, respectively. (b) Two different refinement results are compared: the fit with the $\Gamma 2$ model (red line) explains the intensity of the magnetic Bragg peaks, while $\Gamma 1$ model fails (blue line). (c) Except for the background signal due to the sample environments, the magnetic Bragg pattern indicates that the magnetic structure is independent of temperature down to $0.07 \mathrm{~K}$.

to be magnetically ordered. The estimated weight percentages of the impurity phases are $3.33 \%$ for $\mathrm{PtAl}_{2}$ and $5.21 \%$ for $\mathrm{Al}$, where the Brindley coefficients are set to 1 for all phases [35].

The antiferromagnetic ordering temperature $\left(T_{\mathrm{N}}\right)$ of $\mathrm{CePtAl}_{4} \mathrm{Ge}_{2}$ is $2.3 \mathrm{~K}$, and the saturated magnetic moment is approximately $1.2 \mu_{\mathrm{B}}$ and $0.4 \mu_{\mathrm{B}}$ for the magnetic field of $50 \mathrm{kOe}$ applied along the crystallographic [100] and [001] direction, respectively [32]. Figure 2(a) shows the diffraction pattern measured at $10 \mathrm{~K}$, which is well explained by the hightemperature crystal symmetry, indicating the absence of a structural transition at low temperatures. Figure 2(b) displays the Bragg pattern measured below $T_{\mathrm{N}}$, in which additional Bragg peaks appear due to magnetic scattering. Rietveld refinements show that the pattern is best explained by an ordering wave vector $\boldsymbol{k}=(1.39,0,0.09)$. This ordering wave vector was confirmed by the thermal neutron single-crystal diffraction experiment. The diffraction pattern at $0.07 \mathrm{~K}$, shown in Fig. 2(c), is similar to that at $1.7 \mathrm{~K}$, except for a broad hump at $2 \theta$ from $14^{\circ}$ to $24^{\circ}$ and a peak at $35.9^{\circ}$. All magnetic peaks are well explained by the same $\boldsymbol{k}$, whereas the hump and peak are due to the low-temperature sample
TABLE I. Basis vectors of the Ce atoms in position $3 b(0,0$, $0.5), R \overline{3} m, \boldsymbol{k}=(1.39,0,0.09)$ in $\mathrm{CePtAl}_{4} \mathrm{Ge}_{2}$. Note that $m_{100}, m_{010}$, and $m_{001}$ denote the magnetic moment of the $\mathrm{Ce}$ atom along the crystallographic [100], [010], and [001] directions, respectively.

\begin{tabular}{lccc}
\hline \hline IR & $m_{100}$ & $m_{010}$ & $m_{001}$ \\
\hline$\Gamma_{1}$ & 0 & 1 & 0 \\
$\Gamma_{2}$ & $2 / \sqrt{ } 3$ & $1 / \sqrt{ } 3$ & 0 \\
& 0 & 0 & 1 \\
\hline \hline
\end{tabular}

environment induced by the dilution refrigerator and are independent of temperature (not shown here).

The representational analysis with the magnetic ordering wave vector $\boldsymbol{k}$ and crystal structure yields two onedimensional irreducible representations (IRs) for the Ce site $(3 b)$, which are summarized in Table I. Figures 2(b) and 2(c) show the refinement with the magnetic $\Gamma_{2}$ IR model and the basis vectors $\boldsymbol{v}_{1}=[2,1,0] / \sqrt{ } 3$ and $\boldsymbol{v}_{2}=[0,0,1]$, which matches the intensities of the magnetic Bragg peaks and is in agreement with the in-plane easy-axis anisotropy deduced from the magnetic susceptibility (Fig. 5). This $\Gamma_{2}$ IR allows for solutions with an arbitrary phase between $\boldsymbol{v}_{1}$ and $\boldsymbol{v}_{2}$ which would correspond to cycloidal and helixlike structures. The best refinement of the magnetic Bragg peaks was obtained for a magnetic phase $\phi=0$ and the fit quality was decreased for $\phi=\pi / 2$ (cycloidal or helixlike phase), indicating that the nature of the magnetically ordered state is not cycloidal or helixlike. The ordered magnetic moments, $\boldsymbol{\mu}^{\text {ord }}=$ $\boldsymbol{\mu}^{[210] / \sqrt{ } 3}+\boldsymbol{\mu}^{[001]}$, which are estimated by Rietveld refinements of neutron powder-diffraction pattern are $\boldsymbol{\mu}^{[210] / \sqrt{ } 3}=$ $1.11(4)$ and $\mu^{[001]}=0.12(3) \mu_{\mathrm{B}}$ at the temperature of $1.7 \mathrm{~K}$ and $\boldsymbol{\mu}^{[210] / \sqrt{ } 3}=1.28(7)$ and $\boldsymbol{\mu}^{[001]}=0.12(4) \mu_{\mathrm{B}}$ at $0.07 \mathrm{~K}$. $\mathrm{Ce}$ moments form a ferromagnetic alignment between neighbors along the crystallographic [010] direction, whereas the magnitude of the moments is modulated along the [100] and [001] directions. Further, the moments are aligned antiparallel along the [100] direction and are aligned parallel with respect to the equivalent site in the next chemical unit cell along the [001] direction. This amplitude modulation of the ordered magnetic moments preserves the inversion center of the magnetic structure. Figures 3(a) and 3(b) visualize the magnetic structure, in which $\mathrm{Ce}$ atoms and their magnetic moments are shown by yellow circles and red arrows, respectively. As shown in Fig. 3(a), three layers of antiferromagnetic triangular lattice are stacked along the crystallographic [001] direction and the dashed line indicates one chemical unit cell. Spin directions in the triangular plane are tilted $30^{\circ}$ with respect to the crystallographic [100] direction, whereas the tilting out of the triangular plane is determined by ratio between $\mu^{[001]}$ and $\boldsymbol{\mu}^{[210] / \sqrt{ } 3}$, i.e., $\theta=\tan ^{-1}\left(\boldsymbol{\mu}^{[001]} / \boldsymbol{\mu}^{[210] / \sqrt{ } 3}\right)$. Refinement of the powder pattern reveals that the angle $\theta$ is $5-6$ degrees at 1.7 and $0.07 \mathrm{~K}$. Further measurements, however, are essential to estimate the exact tilting angle and its temperature dependence.

The single-crystal neutron diffraction data in Figs. 3(c) and 3(d) are consistent with the incommensurate ordering wave vector $\boldsymbol{k}=(1.39,0,0.09)$ obtained from the powder diffraction analysis. Figure 3(c) shows two $h$-scan data around 
(a)

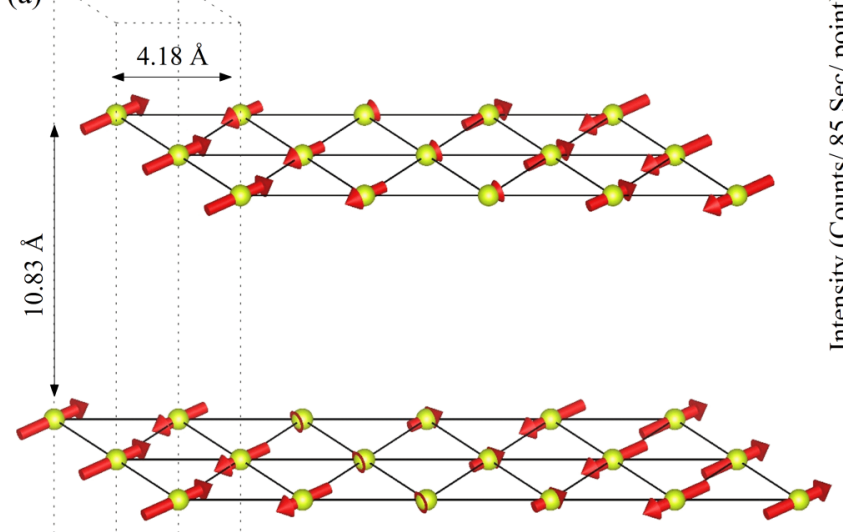

(b)

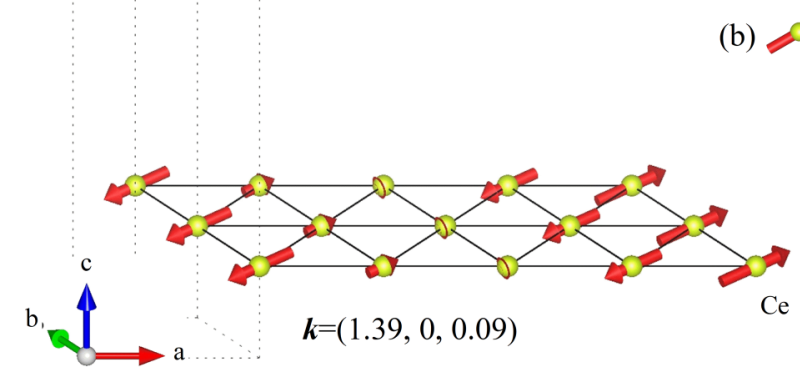

(c)

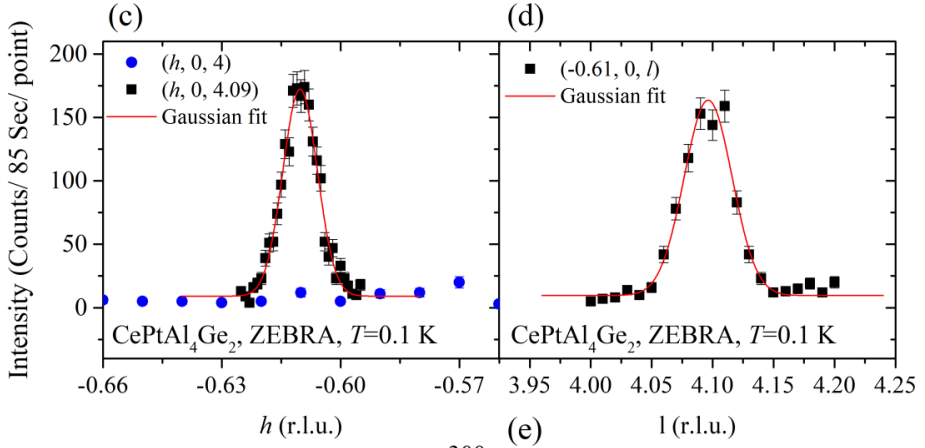

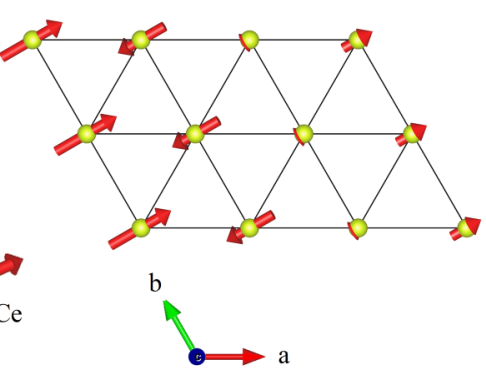

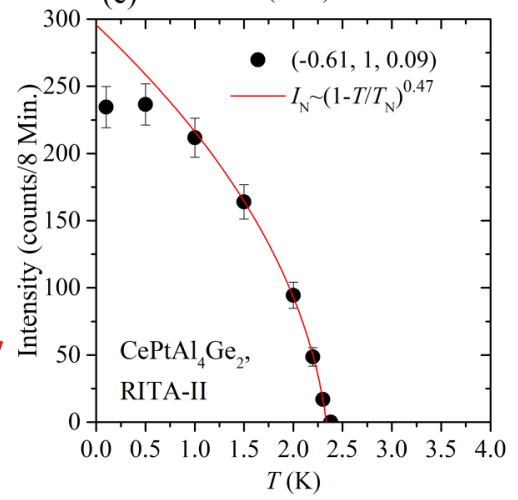

FIG. 3. Magnetic structure and single-crystal neutron diffraction of $\mathrm{CePtAl}_{4} \mathrm{Ge}_{2}$. Magnetic structure with $\boldsymbol{k}=(1.39,0,0.09)$ is sketched in three-dimensional space in (a) and within the Ce plane in (b). The amplitude of the Ce magnetic moments is represented by the length of the arrows. (c) Single-crystal neutron-diffraction intensity at $0.1 \mathrm{~K}$ is plotted as a function of $h$ (reciprocal lattice). The solid line in the inset is a Gaussian function with its center at $h=-0.61$, where the peak is only observed when the ordering wave vector contains incommensurate factor along the reciprocal $l$ direction. (d) A magnetic Bragg peak at $0.1 \mathrm{~K}$ is observed by $l$ scan of $(-0.61,0, l)$, where the peak position is determined to be $l=4.09$ from a Gaussian fitting. (e) Temperature dependence of the neutron intensity of the magnetic Bragg peak shows a critical behavior with a temperature exponent of $0.47 \pm 0.04$ and a critical temperature of $2.38 \mathrm{~K}$.

$h=-0.61$, where blue circles represent the $h$ scan of $(h, 0$, 4) for $l=0$ and black squares describe the $h$ scan of $(h, 0$, 4.09) for $l \neq 0$. A magnetic Bragg peak is only observed in the $(h, 0,4.09)$ scan, showing the existence of small $l$ value in $\boldsymbol{k}$. A Gaussian fit of the Bragg peak (red solid line) gives the center value of $h=-0.61$, which is consistent with the $h$ value of 1.39 from the refinement on the powder pattern. Figure 3(d) describes an $l$ scan of $(-0.61,0, l)$, in which a Gaussian fit best explains the peak at $l=0.09 \pm 0.005$. The magnetic Bragg position of $(-0.61,0,4.09)$ is equivalent to adding $\boldsymbol{k}$ to an allowed nuclear Bragg position $(-2,0$, $4)$, i.e., $(-0.61,0,4.09)=(-2,0,4)+\boldsymbol{k}$. The temperature dependence of the neutron intensity $\left(I_{\mathrm{N}}\right)$ of the magnetic Bragg peak is shown in Fig. 3(e), showing that it becomes nonzero below $2.38 \mathrm{~K}$, in agreement with $T_{\mathrm{N}}$ determined from thermodynamic and transport measurements [32]. The critical exponent $(\beta)$ of the AFM ordered moment deduced from the fit, $I_{\mathrm{N}} \sim\left(\mu^{\text {ord }}\right)^{2} \sim\left(1-T / T_{\mathrm{N}}\right)^{2 \beta}$, is $0.237 \pm 0.021$, which is in good agreement with the value of 0.23 estimated from the two-dimensional $X Y$ magnet [38,39]. The two-dimensional $X Y$-type ordered moments are consistent with the magnetic structure of $\mathrm{CePtAl}_{4} \mathrm{Ge}_{2}$ as shown in Fig. 3(a).

Figure 4 shows the results of the inelastic neutronscattering experiments on a powder sample. The neutron intensity as a function of transferred energy $\left(E_{\mathrm{T}}=E_{\mathrm{i}}-E_{\mathrm{f}}\right)$ was measured at 10 and $120 \mathrm{~K}$ at various $\mathbf{Q}$ values, where $\mathbf{Q}=\mathbf{k}_{\mathrm{i}}-\mathbf{k}_{\mathrm{f}}$ is the momentum transfer. All spectra show a peak due to magnetic excitations around $E_{\mathrm{T}}=14.5 \mathrm{meV}$ and

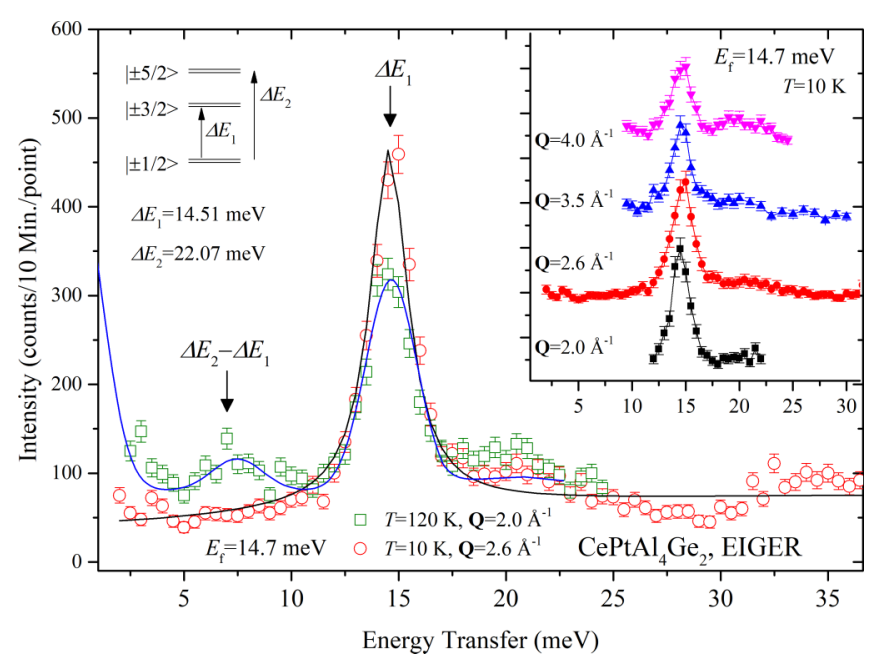

FIG. 4. Inelastic neutron spectra of $\mathrm{CePtAl}_{4} \mathrm{Ge}_{2}$. Inelastic neutron scattering is measured at 10 and $120 \mathrm{~K}$, which reveals a magnetic excitation around $14.5 \mathrm{meV}$. Inset shows the intensity of this peak collected at various $\mathbf{Q}$ values at $10 \mathrm{~K}$. The least-squares fit (black line) of the inelastic scattering data (red circles) with $\mathbf{Q}=2.6 \AA^{-1}$ by a Gaussian function is centered at $14.51 \mathrm{meV}$, which was ascribed to the crystal-field splitting between the ground state $\left(\left|j_{z}=1 / 2\right\rangle\right)$ and first excited $\left(\left|j_{\mathrm{z}}=3 / 2\right\rangle\right)$ doublets. The spectrum measured at $120 \mathrm{~K}$ (green squares in the main panel) is well fitted by the same CEF parameters, and a peak at $7.5 \mathrm{meV}$ is consistent with the CEF excitation from the $\left|j_{\mathrm{z}}=3 / 2\right\rangle$ to the $\left|j_{\mathrm{z}}=5 / 2\right\rangle$ doublets. 

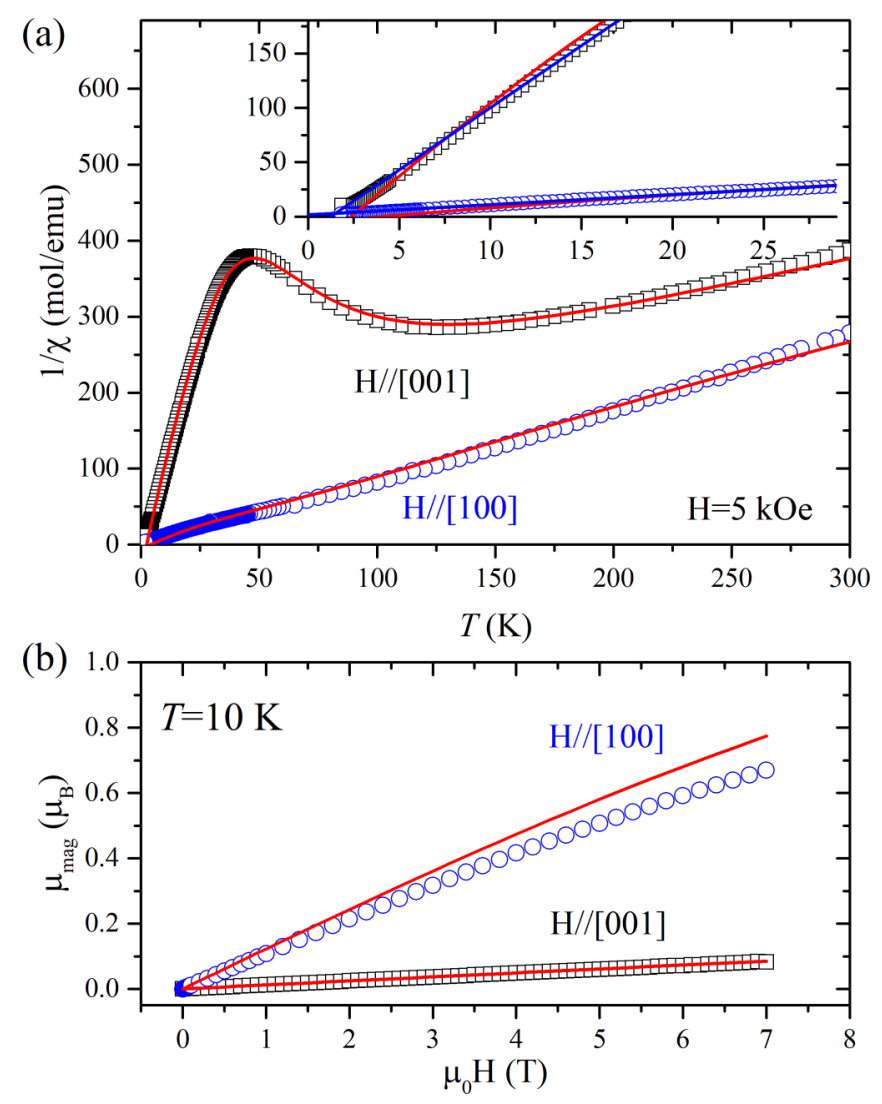

FIG. 5. Temperature and field dependence of magnetization of $\mathrm{CePtAl}_{4} \mathrm{Ge}_{2}$. The inverse of the magnetic susceptibility, $1 / \chi$, is shown as a function of temperature in (a) and the magnetic moment as a function of magnetic field is displayed in (b) for two different field orientations of $H \|[100]$ (blue circles) and $H \|[001]$ (black squares). Solid lines are the least-squares fittings by the effective Hamiltonian, $H_{\text {eff }}$, that includes contributions from CEF, Zeeman, and anisotropic exchange interactions (see the text for details). The inset shows the low-temperature regime of Fig. 5(a) and the least-squares fits (blue line) of $1 /\left(\chi-\chi_{0}\right)=\left(T-\theta_{\mathrm{CW}}\right) / C$ in the temperatures below $20 \mathrm{~K}$. The $\chi_{0}$ values are 0.00067 and 0.00058 emu/mol for $H \|[100]$ and $H \|[001]$, respectively.

the inset shows that its peak intensity is gradually suppressed as $\mathbf{Q}$ increases, which can be explained by the square of the magnetic form factor of $\mathrm{Ce}^{3+}$ ions. At an elevated temperature of $120 \mathrm{~K}$, this Gaussian peak becomes weaker. The inelastic neutron intensity with $\mathbf{Q}=2.6 \AA^{-1}$, as shown in Fig. 4 , is best described by the Gaussian distribution with its center at $E_{\mathrm{T}}=14.51 \mathrm{meV}$. Taken together, these $\mathbf{Q}$ and temperature dependence of the inelastic neutron-scattering intensity indicate that the excitation at $14.51 \mathrm{meV}$ is the CEF first excited state of the $\mathrm{Ce}^{3+}$ multiplet. We note that there is a small hump around $E_{\mathrm{T}}=20 \mathrm{meV}$, whose origin has yet to be clarified through additional measurements.

\section{DISCUSSION}

Figures 5(a) and 5(b) display the temperature dependence of the inverse of the magnetic susceptibility $(\chi)$ and the magnetic field dependence of the magnetic moment, $\mu_{\mathrm{mag}}(H)$, respectively. The solid lines are the least-squares fits obtained from the MANTID program which contains crystalline electric field (CEF) effects [40]. Ce sites in $\mathrm{CePtAl} \mathrm{Ge}_{2}$ occupy the position $(0,0,0.5)$ with local trigonal symmetry $D_{3 d}$. As a result, the CEF potential $\left(H_{\mathrm{CEF}}\right)$ can be described by the following Hamiltonian: $\mathrm{H}_{\mathrm{CEF}}=\mathrm{B}_{2}{ }^{0} \mathrm{O}_{2}{ }^{0}+\mathrm{B}_{4}{ }^{0} \mathrm{O}_{4}{ }^{0}+\mathrm{B}_{4}{ }^{3} \mathrm{O}_{4}{ }^{3}$, where $B_{l}{ }^{m}$ and $O_{l}{ }^{m}$ are CEF parameters and Stevens operators, respectively $[41,42]$. Based on the single-ion point-charge model, initial $B_{l}{ }^{m}$ parameters were obtained by using the MANTID package with six $\mathrm{Ge}^{4+}$ ions located in the nearest neighbors (NNs). When the magnetic easy axis is within the $x y$ plane, $B_{2}{ }^{0}$ should be positive $[43,44]$. If $B_{2}{ }^{0}$ is a positive number, only six $\mathrm{Ge}^{4+}$ ions should be considered because $B_{2}{ }^{0}$ becomes negative when six $\mathrm{Al}^{3+}$ ions in the nextnearest neighbors (NNNs) are additionally included. Pointcharge model simulation with the $\mathrm{NN} \mathrm{Ge}^{4+}$ ions produces the best results when $B_{l}{ }^{m}$ parameters are $B_{2}{ }^{0}=6.63 \mathrm{meV}$, $B_{4}{ }^{0}=0.13 \mathrm{meV}$, and $B_{4}{ }^{3}=-3.49 \mathrm{meV}$. Based on these initial CEF parameters, simulations on the magnetization curves were performed by the following effective Hamiltonian: $H_{\mathrm{eff}}=H_{\mathrm{CEF}}+g_{\mathrm{J}} \mu_{\mathrm{B}} \Sigma_{(i=x, z)} j^{i}\left(H_{\text {ext }}^{i}+H_{m f}^{i}\right)$, where the contributions from CEF, Zeeman effect, and mean-field-type anisotropic exchange interaction are included as the first, second, and third term, respectively. Here $g_{\mathrm{J}}$ is $6 / 7$ due to the $\mathrm{Ce}^{3+}, \mu_{\mathrm{B}}$ is the Bohr magneton, $j^{i}$ is the total angular magnetic moment for the two different directions $(i=x, z)$, $H_{\text {ext }}$ is the external applied magnetic field, and $H_{m f}$ is the mean-field anisotropic molecular field which is described by $H_{m f}=\lambda^{i}\left\langle\boldsymbol{M}^{i}\right\rangle=g_{\mathrm{J}} \mu_{\mathrm{B}} \lambda^{i}\left\langle j^{i}\right\rangle$.

Figures 5(a) and 5(b) describe the analysis of the magnetization curves for both field directions of $H \|[001]$ and $H \|[100]$ by using the effective Hamiltonian $H_{\text {eff }}$. The results of CEF analysis are summarized in the Table II. The neutron spectra measured at 10 and $120 \mathrm{~K}$ are well explained as shown in Fig. 4. The first excited level is close to $14.5 \mathrm{meV}$, which is the peak position in the inelastic neutron-scattering measurements. The calculated excitation energy for the second excited state $\left(\Delta E_{2}\right)$ is $22.07 \mathrm{meV}$. Even though a small hump is observed at a similar value in the inelastic neutron spectra in Fig. 4, it may not be related to the CEF magnetic excitation because the possibility of the second excitation is zero due to the selection rule, i.e., $\left\langle 1 / 2\left|J_{x, y, z}\right| 5 / 2\right\rangle=0$. A broad peak observed at $7.5 \mathrm{meV}$ in the inelastic neutron spectrum at $120 \mathrm{~K}$ (green squares), on the other hand, could be ascribed to the CEF excitation from $\left|j_{z}=3 / 2\right\rangle$ to $\left|j_{z}=5 / 2\right\rangle$ doublets because the position of the additional peak is consistent with the energy difference between $\left|j_{z}=3 / 2\right\rangle$ and $\left|j_{z}=5 / 2\right\rangle$ (22.07 $\left.-14.51 \mathrm{meV}=\Delta E_{2}-\Delta E_{1}\right)$. The saturated magnetic ordered moment along the crystallographic [001] and [100] axis is expressed by $g_{\mathrm{J}} \mu_{\mathrm{B}}\left\langle\Gamma\left|J_{z}\right| \Gamma\right\rangle$ and $g_{\mathrm{J}} \mu_{\mathrm{B}}\left\langle\Gamma\left|J_{x}\right| \Gamma\right\rangle$ where $\Gamma$ is the crystal-field ground state. The calculated magnetic moments from the obtained ground state, $\left|j_{\mathrm{z}}=1 / 2\right\rangle$, are $0.43 \mu_{\mathrm{B}}$ and $1.25 \mu_{\mathrm{B}}$, respectively, which are comparable to the saturated magnetic moments of $0.4 \mu_{\mathrm{B}}$ and $1.2 \mu_{\mathrm{B}}$, respectively [32]. We note that the Curie-Weiss temperatures $\left(\theta_{\mathrm{CW}}\right)$ deduced from the susceptibility at low temperatures are $2.78 \pm 0.03$ and $0.10 \pm 0.01 \mathrm{~K}$ for $H \|[001]$ and $H \|[100]$, respectively, indicating that the competition between ferromagnetic and antiferromagnetic interactions could be a source of the magnetic frustration within the triangular plane. 
TABLE II. CEF analysis results of $\mathrm{CePtAl}_{4} \mathrm{Ge}_{2}$.

\begin{tabular}{|c|c|c|c|c|c|c|}
\hline & \multicolumn{2}{|c|}{$B_{2}{ }^{0}(\mathrm{meV})$} & \multicolumn{2}{|c|}{$B_{4}{ }^{0}(\mathrm{meV})$} & \multicolumn{2}{|c|}{$B_{4}{ }^{3}(\mathrm{meV})$} \\
\hline \multirow[t]{2}{*}{$\lambda(\mathrm{mol} / \mathrm{emu})$} & $\lambda^{x}$ & $\lambda^{z}$ & & $\chi_{0}(\mathrm{emu} / \mathrm{mol})$ & $\chi_{0}^{x}$ & $\chi_{0}^{z}$ \\
\hline & $6.4 \pm 0.3$ & $34.5 \pm 0.3$ & & & 0.00067 & 0.00058 \\
\hline$\Delta E(\mathrm{meV})$ & $|5 / 2\rangle$ & $|3 / 2\rangle$ & $|1 / 2\rangle$ & $|-1 / 2\rangle$ & $|-3 / 2\rangle$ & $|-5 / 2\rangle$ \\
\hline 22.07 & 0 & 0 & $O(-6)$ & 0 & 0 & $\sim 1$ \\
\hline 22.07 & $\sim 1$ & 0 & 0 & $O(-6)$ & 0 & 0 \\
\hline 14.51 & 0 & 0 & 0 & 0 & -1 & 0 \\
\hline 14.51 & 0 & -1 & 0 & 0 & 0 & 0 \\
\hline
\end{tabular}

Magnetic moments of $\mathrm{CePtAl}_{4} \mathrm{Ge}_{2}$ that form a triangular lattice become antiferromagnetically ordered below $2.3 \mathrm{~K}$, where neighbors are aligned ferromagnetically along the [010] direction and are modulated along the [100] and [001] directions. The peculiar AFM magnetic structure observed in our experiment with incommensurate magnetic ordering vector $\boldsymbol{k}=(1.39,0,0.09)$ within a triangular lattice is rare. Mixed magnetic structure with amplitude modulation has been reported in the $f$-orbital frustrated magnetic systems with Kondo effects and magnetic frustration [7,8,13]. The distorted kagome lattice $\mathrm{CePdAl}$, where $1 / 3$ of Ce moments are disordered, while $2 / 3$ of them are ordered with incommensurate $\boldsymbol{k}=(0.5,0, \sim 0.35)$ in AFM state, the moment are modulating along the [001] direction [7,45,46]. Similar mixed magnetic structure in the AFM state was also reported in the U-based hexagonal frustrated magnet $\mathrm{UNi}_{4} \mathrm{~B}$, where $1 / 3$ of $U$ moments remain paramagnetic, while $2 / 3$ of them are ordered toroidally within the hexagonal plane [12]. The AFM compound $\mathrm{CePtAl}_{4} \mathrm{Ge}_{2}$, where amplitude modulation of the spin chains was observed along the crystalline [100] direction, will provide a rare opportunity to study the interplay between Kondo effects and magnetic frustration. It will be a challenge to determine the role played by such factors as competition between FM and AFM interactions, RKKY and Kondo interactions, and the triangular crystalline structure in producing magnetic frustration in this compound.

\section{SUMMARY}

We performed elastic and inelastic neutron-scattering experiments and a CEF study of the triangular antiferromagnet $\mathrm{CePtAl}_{4} \mathrm{Ge}_{2}$. The Rietveld analysis of the neutron powder pattern and the direct observation of the magnetic Bragg peaks using thermal neutron single-crystal diffraction showed that the AFM ordered state is an amplitude incommensurate structure modulated by the ordering wave vector of $(1.39,0$, 0.09). Here, neighbors are aligned ferromagnetically along the [010] direction and the amplitude of magnetic moments oscillates periodically along the [100], and [001] directions, suggesting an intricate interplay between magnetic frustration, Kondo effect, and RKKY interactions. When combined with the inelastic neutron-scattering results, the temperature and field dependence of the magnetization of $\mathrm{CePtAl}_{4} \mathrm{Ge}_{2}$ is consistent with a doublet ground state with a dominant $\left|j_{\mathrm{z}}=1 / 2\right\rangle$ character and a first excited doublet $\left|j_{\mathrm{z}}=3 / 2\right\rangle$ at $14.5 \mathrm{meV}$.

\section{ACKNOWLEDGMENTS}

This work was supported by a National Research Foundation (NRF) of Korea grant funded by the Korean Ministry of Science and ICT (Grant No. 2012R1A3A2048816). Work at Paul Scherrer Institut in Switzerland was done as a fellow in the "Young Researchers Exchange Programme between Korea and Switzerland" under the "Korean-Swiss Science and Technology Programme 2016-2017." Neutronscattering experiments were carried out at the continuous spallation neutron source SINQ at the Paul Scherrer Institut at Villigen PSI in Switzerland. Magnetization measurements were performed at the Laboratory for Multiscale Materials Experiments of Paul Scherrer Institute. Work at Los Alamos National Laboratory was performed under the auspices of the U.S. Department of Energy, Office of Basic Energy Sciences, Division of Materials Sciences and Engineering.
[1] C. Lacroix, J. Phys. Soc. Jpn. 79, 011008 (2010).

[2] G. H. Wannier, Phys. Rev. 79, 357 (1950).

[3] J. Vannimenus and G. Toulouse, J. Phys. C 10, L537 (1977).

[4] J. Villain, J. Phys. C 10, 1717 (1977).
[5] H. T. Diep, Frustrated Spin Systems (World Scientific, Singapore, 2004).

[6] L. Balents, Nature (London) 464, 199 (2010).

[7] A. Dönni, G. Ehlers, H. Maletta, P. Fischer, H. Kitazawa, and M. Zolliker, J. Phys.: Condens. Matter 8, 11213 (1996). 
[8] C. Lacroix, B. Canals, and M. D. Núñez-Regueiro, Phys. Rev. Lett. 77, 5126 (1996).

[9] J.-X. Zhu, I. Martin, and A. R. Bishop, Phys. Rev. Lett. 100, 236403 (2008).

[10] S. Julian and H.-Y. Kee, Phys. Can. 68, 95 (2012).

[11] S. Lucas, K. Grube, C.-L. Huang, A. Sakai, S. Wunderlich, E. L. Green, J. Wosnitza, V. Fritch, P. Gegenwart, O. Stockert, and H. V. Löhneysen, Phys. Rev. Lett. 118, 107204 (2017).

[12] S. A. M. Mentink, A. Drost, G. J. Nieuwenhuys, E. Frikkee, A. A. Menovsky, and J. A. Mydosh, Phys. Rev. Lett. 73, 1031 (1994).

[13] P. Bonville, M. Rams, K. Królas, J. P. Sanchez, P. C. Canfield, O. Trovarelli, and C. Geibel, Eur. Phys. J. B 55, 77 (2007).

[14] B. Fåk, C. Rüegg, P. G. Niklowitz, D. F. McMorrow, P. C. Canfield, S. L. Bud'ko, Y. Janssen, and K. Habicht, Phys. B (Amsterdam, Neth.) 378-380, 669 (2006).

[15] T. Kurumaji, T. Nakajima, M. Hirschberger, A. Kikkawa, Y. Yamasaki, H. Sagayama, H. Nakao, Y. Taguchi, T.-h. Arima, and Y. Tokura, Science 365, 914 (2019).

[16] F. Ronning, T. Helm, K. R. shirer, M. D. Bachmann, L. Balicas, M. K. Chan, B. J. Ramshaw, R. D. McDonald, F. F. Balakirev, M. Jaime, E. D. Bauer, and P. J. W. Moll, Nature (London) 548, 313 (2017).

[17] D. M. Fobes, S. Zhang, S.-Z. Lin, Pinaki Das, N. J. Ghimire, E. D. Bauer, J. D. Thompson, L. W. Harriger, G. Ehlers, A. Podlesnyak, R. I. Bewley, A. Sazonov, V. Hutanu, F. Ronning, C. D. Batista, and M. Janoschek, Nat. Phys. 14, 456 (2018).

[18] P. F. S. Rosa, S. M. Thomas, F. F. Balakirev, E. D. Bauer, R. M. Fernandes, J. D. Thompson, F. Ronning, and M. Jaime, Phys. Rev. Lett. 122, 016402 (2019).

[19] K. Mochidzuki, Y. Shimizu, A. Kondo, S. Nakamura, S. Kittaka, Y. Kono, T. Sakakibara, Y. Ikeda, Y. Isikawa, and K. Kindo, J. Phys. Soc. Jpn. 86, 034709 (2017).

[20] H. Zhao, J. Zhang, M. Lyu, S. Bachus, Y. Tokiwa, P. Gegenwart, S. Zhang, J. Cheng, Y.-f. Yang, G. Chen, Y. Isikawa, Q. Si, F. Steglich, and P. Sun, Nat. Phys. 15, 1261 (2019).

[21] V. Fritsch, N. Bagrets, G. Goll, W. Kittler, M. J. Wolf, K. Grube, C. L. Huang, and H. v. Löhneysen, Phys. Rev. B 89, 054416 (2014).

[22] J. Zhang, H. Zhao, M. Lv, S. Hu, Y. Isikawa, Y. F. Yang, Q. Si, F. Steglich, and P. Sun, Phys. Rev. B 97, 235117 (2018).

[23] Y. Tokiwa, M. Garst, P. Gegenwart, S. L. Bud'ko, and P. C. Canfield, Phys. Rev. Lett. 111, 116401 (2013).

[24] E. Mun, S. L. Bud'ko, and P. C. Canfield, Phys. Rev. B 82, 174403 (2010).

[25] K. Sengupta, M. K. Forthaus, H. Kubo, K. Katoh, K. Umeo, T. Takabatake, and M. M. Abd-Elmeguid, Phys. Rev. B 81, 125129 (2010).

[26] Y. Tokiwa, C. Stingl, M.-S. Kim, T. Takabatake, and P. Gegenwart, Sci. Adv. 1, e1500001 (2015).

[27] R. Küchler, C. Stingl, Y. Tokiwa, M. S. Kim, T. Takabatake, and P. Gegenwart, Phys. Rev. B 96, 241110(R) (2017).
[28] C. L. Yang, S. Tsuda, K. Umeo, Y. Yamane, T. Onimaru, T. Takabatake, N. Kikugawa, T. Terashima, and S. Uji, Phys. Rev. B 96, 045139 (2017).

[29] P. Coleman and A. H. Nevidomskyy, J. Low Temp. Phys. 161, 182 (2010).

[30] S. Burdin, D. R. Grempel, and A. Georges, Phys. Rev. B 66, 045111 (2002).

[31] Q. Si, J. H. Pixley, E. Nica, S. J. Yamamoto, P. Goswami, R. Yu, and S. Kirchner, J. Phys. Soc. Jpn. 83, 061005 (2014).

[32] S. Shin, P. F. S. Rosa, F. Ronning, J. D. Thompson, B. L. Scott, S. Lee, H. Jang, S.-G. Jung, E. Yun, H. Lee, E. D. Bauer, and T. Park, J. Alloys Compd. 738, 550 (2018).

[33] P. Fischer, G. Frey, M. Koch, M. KoK nnecke, V. Pomjakushin, J. Schefer, R. Thut, N. Schlumpf, R. Bürge, U. Greuter, S. Bondt, and E. Berruyer, Phys. B (Amsterdam, Neth.) 276-278, 146 (2000).

[34] J. Schefer, P. Fischer, H. Heer, A. Isacson, M. Koch, and R. Thut, Nucl. Instrum. Methods Phys. Res. A 288, 477 (1990).

[35] J. Rodríguez-Carvajal, Phys. B (Amsterdam, Neth.) 192, 55 (1993).

[36] M. I. Aroyo, J. M. Perez-Mato, D. Orobengoa, E. Tasci, G. De La Flor, and A. Kirov, Bulg. Chem. Commun. 43, 183 (2011).

[37] In order to obtain the large signal-to-background ratio, a larger single crystal was used to measure the magnetic susceptibility data. The $1 / \chi(T)$ in Fig. 5 exhibits the Curie-Weiss behavior at a wider temperature range than that of Ref. [32] at high temperatures. As a result, the $\chi_{0}$ values extracted here are two times smaller than the values from Ref. [32]. Erratum has been submitted to the Journal of Alloys and Compounds to take into account the change made in this manuscript.

[38] S. T. Bramwell and P. C. W. Holdsworth, J. Appl. Phys. 73, 6096 (1993).

[39] S. T. Bramwell and P. C. W. Holdsworth, J. Phys.: Condens. Matter 5, L53 (1993).

[40] O. Arnold, J. C. Bilheux, J. M. Borreguero, A. Buts, S. I. Campbell, and L. Chapon, M. Doucet, N. Draper, R. Ferraz Leal, M. A. Gigg, V. E. Lynch, A. Markvardsen, D. J. Mikkelson, R. L. Mikkelson, R. Miller, K. Palmen, P. Parker, G. Passos, T. G. Perring, P. F. Peterson et al., Nucl. Instrum. Methods Phys. Res. 764, 156 (2014).

[41] K. W. H. Stevens, Proc. Phys. Soc. A 65, 209 (1952).

[42] F. Seitz and D. Turnbull, Solid State Physics (Academic, New York, 1964).

[43] N. V. Hieu, T. Takeuchi, H. Shishido, C. Tonohiro, T. Yamada, H. Nakashima, K. Sugiyama, R. Settai, T. D. Matsuda, Y. Haga, M. Hagiwara, K. Kindo, S. Araki, Y. Nozue, and Y. Onuki, J. Phys. Soc. Jpn. 76, 064702 (2007).

[44] P. F. S. Rosa, R. J. Bourg, C. B. R. Jesus, P. G. Pagliuso, and Z. Fisk, Phys. Rev. B 92, 134421 (2015).

[45] L. Keller, A. Dönni, H. Kitazawa, and B. van den Brandt, Appl. Phys. A 74, s686 (2002).

[46] V. Fritsch, S. Lucas, Z. Huesges, A. Sakai, W. Kittler, C. Taubenheim, S. Woitschach, B. Pedersen, K. Grube, B. Schmidt, P. Gegenwart, O. Stockert, and H. v. Löhneysen, J. Phys.: Conf. Ser. 807, 032003 (2017). 